\title{
Tuning in to art: A predictive processing account of negative emotion in art (commentary to Menninghaus et al. BBS)
}

\author{
Sander Van de Cruys $^{1}$, \\ Rebecca Chamberlain ${ }^{1,2}$, \\ Johan Wagemans ${ }^{1}$ \\ ${ }^{1}$ Brain \& Cognition Unit, KU Leuven, Belgium \\ ${ }^{2}$ Department of Psychology, Goldsmiths, University of London, UK
}

\begin{abstract}
We use the example of art-derived solace to discuss a broader mechanism by which negative affect is instrumental in creating positive appreciation of artworks. Based on the theory of predictive processing, we argue that increasing attunement or reduction of prediction errors, which implies increasing validation of the agent('s models), is experienced as positive, even if the artwork's content is negative.
\end{abstract}

We commend Menninghaus et al. for their thorough and insightful treatment of negative affect in aesthetic experience. As they illustrate, negative affect is ubiquitous and non-accidental in art, so the question of why people seek out negative affect in art is a fascinating and important one. While Menninghaus et al. highlight several possible explanations, we think they leave underexposed the central role of consolation in art appreciation.

Very often, people read novels to find solace concerning every-day or existential uncertainties and anxieties. They watch films and television series to remind themselves they are not alone in their failings, inner conflicts or even idiosyncratic pleasures. Even if no concrete solutions for sorrows are offered in the artwork, the mere discovery of similar affective dynamics validates the existence of the perceivers and the cognitive/affective schemata with which they experience (and navigate through) the world. Although the content of these dynamics is often negative, the process is one of increasing attunement between artwork and perceiver.

On the face of it, this could be considered a kind of empathic affect (Jackendoff \& Lerdahl, 2006) where the perceiver empathizes with the creator of the artwork or the performer of the dance or music act. But the point is not that we have compassion with themes or people in the artwork and subsequently, as a kind of meta-emotion, feel good about this pro-social response (what Menninghaus et al. do discuss but dismiss). More often, the "empathic" reflection is one of more direct attunement with the artwork, for example with the structural aspects of the music that convey sadness, joy,... by way of modulation of tone, tempo, timbre, melodic contour, etc. 
The "language" by which the musician or painter's technique allows us to "empathize" with the work directly is insufficiently understood but probably relates to natural expressive speech or expressive behavior (Freedberg \& Gallese, 2007; Jackendoff \& Lerdahl, 2006). What is clear, is that we have implicit generative models of how particular artistic outputs are created (as for any other perceptual inputs; e.g., Kersten, Mamassian, \& Yuille, 2004). Perceiving those outputs is inferring their hidden causes, which includes not only specific patterns of motor behavior (e.g. strumming a guitar, "stroking" a canvas), but also the (hierarchically) deeper causes, in terms of conceptual and emotional/motivational states. Meanwhile, the content of the artwork can set the context for a better alignment of the models of the perceiver and those implied in the work. The commonality in generative models that allows for synchronization of state dynamics has recently been used to study mutual understanding in social interactions, where a shared conceptual space enables agents to predict each other (Friston \& Frith, 2015). However, the analysis here suggests this form of alignment is not limited to explicit social interactions.

The exceptional thing about art and music is that they often invoke affective narratives that are rarely explicitly articulated but nonetheless find resonance in the affective models of perceivers. Here, art has the potential to resolve inner conflicts or ambiguities through attunement, by confirming models that allow seemingly contradicting states to coexist, or by validating one model over the other.

But good artworks will also (temporarily) obstruct alignment, and this obstruction is often conducive to appreciation. To understand this we can turn to predictive processing as an account that formalizes the mental mechanics of uncertainty reduction. This approach holds that our brain is continually attempting to minimize the prediction errors that reflect the mismatch between its hierarchical models (that we use to predict/interpret inputs) and the actual inputs from our senses (Clark, 2013; Friston, 2010). We previously hypothesized that the transition from a state of higher uncertainty to a state of lower uncertainty (i.e. an active reduction of prediction errors) is experienced as pleasurable, and that this may help to explain our aesthetic experiences, even for 'static' artworks (Van de Cruys \& Wagemans, 2011). Hence, the increasing attunement of models and world (perceptual inputs) yields positive affect (again, even if the content of the models/inputs is about negative events). So with regard to emotional effects, the dynamics of attunement are key. If violations of expectancies are the norm in art, this is because it allows viewers to make greater progress in reducing those "errors" on conceptual or perceptual levels.

If we extend the view above and characterize negative affect as a state of rapidly increasing uncertainty (i.e., the opposite of positive affect), then negative affect is indeed regularly present in our experience with art -in a formal way, not only in terms of negative content. The question of why we seek out negative affect in art then becomes very closely related to the much debated one about why we sometimes seek out uncertainty. One way out, within a predictive processing framework, is to argue that we are intrinsically motivated to seek predictive progress (Gottlieb, Oudeyer, Lopes, \& Baranes, 2013) which necessarily urges us to venture out of predictable zones. However, we do not do this haphazardly; we seem to contextually learn (meta)predictions on prediction error reduction rates (Van de Cruys, 2017). Those 
expectations on the reducibility of errors can function as implicit appraisals of coping potential that are so important in our engagement with art (Silvia, 2005).

In sum, while we started out describing the important role of solace in art, we came to see it as just an example of broader mechanism by which negative affect is instrumental in creating positive appreciation of artworks. The presented account may serve as an explanation for the "mood congruency effect" (Derryberry, 1988), in which perceivers seek out artworks that correspond with their current or desired mood state (e.g. sad music on a funeral). The view also has some affinity with the processing fluency account, but it adds a process account and emphasizes the temporal dynamics in fluency (cf. relative fluency; Wänke \& Hansen, 2015). We end up with a view in which "being moved by" art is an almost literal consequence of moving through the prediction error gradients.

\section{REFERENCES}

Clark, A. (2013). Whatever next? Predictive brains, situated agents, and the future of cognitive science. The Behavioral and Brain Sciences, 36(03), 181-204.

https://doi.org/10.1017/S0140525X12000477

Derryberry, D. (1988). Emotional influences on evaluative judgments: Roles of arousal, attention, and spreading activation. Motivation and Emotion, 12(1), 23-55. https://doi.org/10.1007/BF00992471

Freedberg, D., \& Gallese, V. (2007). Motion, emotion and empathy in esthetic experience. Trends in Cognitive Sciences, 11(5), 197-203. https://doi.org/10.1016/j.tics.2007.02.003

Friston, K. J. (2010). The free-energy principle: a unified brain theory? Nature Reviews.

Neuroscience, 11(2), 127-138. https://doi.org/10.1038/nrn2787

Friston, K. J., \& Frith, C. D. (2015). Active inference, communication and hermeneutics. Cortex; $a$ Journal Devoted to the Study of the Nervous System and Behavior, 68, 129-143. https://doi.org/10.1016/j.cortex.2015.03.025

Gottlieb, J., Oudeyer, P.-Y., Lopes, M., \& Baranes, A. (2013). Information-seeking, curiosity, and attention: computational and neural mechanisms. Trends in Cognitive Sciences, 17(11), 585-593. https://doi.org/10.1016/j.tics.2013.09.001 
Jackendoff, R., \& Lerdahl, F. (2006). The capacity for music: What is it, and what's special about it? Cognition, 100(1), 33-72. https://doi.org/10.1016/j.cognition.2005.11.005

Kersten, D., Mamassian, P., \& Yuille, A. (2004). Object perception as Bayesian inference. Annual Review of Psychology, 55, 271-304.

https://doi.org/10.1146/annurev.psych.55.090902.142005

Silvia, P. J. (2005). Emotional responses to art: From collation and arousal to cognition and emotion. Review of General Psychology, 9(4), 342. Retrieved from https://pdfs.semanticscholar.org/a87a/95da82793dfe51b6f0a6d9d52c1c5d428f7c.pdf

Van de Cruys, S. (2017). Affective Value in the Predictive Mind. In T. K. Metzinger \& W. Wiese (Eds.), Philosophy and Predictive Processing. Frankfurt am Main: MIND Group. https://doi.org/10.15502/9783958573253

Van de Cruys, S., \& Wagemans, J. (2011). Putting reward in art: A tentative prediction error account of visual art. I-Perception, 2(9), 1035-1062. https://doi.org/10.1068/i0466aap

Wänke, M., \& Hansen, J. (2015). Relative Processing Fluency. Current Directions in Psychological Science, 24(3), 195-199. https://doi.org/10.1177/0963721414561766 